\title{
Front Matter: Volume 9274
}

, "Front Matter: Volume 9274," Proc. SPIE 9274, Advanced Sensor Systems and Applications VI, 927401 (12 December 2014); doi: 10.1117/12.2181316

SPIE. Event: SPIE/COS Photonics Asia, 2014, Beijing, China 


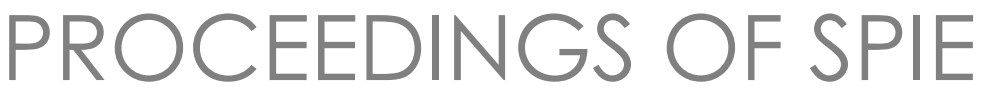

\title{
Advanced Sensor Systems and Applications VI
}

\author{
Tiegen Liu \\ Shibin Jiang \\ Niels Neumann \\ Editors
}

\section{9-11 October 2014 Beijing, China}

Sponsored by

SPIE

COS-Chinese Optical Society

\section{Cooperating Organizations}

Tsinghua University (China) • Peking University (China) • University of Science and Technology of China (China) • Zhejiang University (China) • Tianjin University (China) • Beijing Institute of Technology (China) • Beijing University of Posts and Telecommunications (China) - Nankai University (China) - Changchun University of Science and Technology (China) • University of Shanghai for Science and Technology (China) • Capital Normal University (China) Huazhong University of Science and Technology (China) • Beijing Jiaotong University (China) • Shanghai Institute of Optics and Fine Mechanics (China) - Changchun Institute of Optics and Fine Mechanics (China) • Institute of Semiconductors (China) • Institute of Optics and Electronics (China) • Institute of Physics (China) • Shanghai Institute of Technical Physics (China) - China Instrument and Control Society (China) - Optoelectronics Technology Committee, COS (China) • SPIE National Committee in China (China) • Optical Society of Japan (Japan) • Optical Society of Korea (Korea, Republic of) - The Australian Optical Society (Australia) - Optics and Photonics Society of Singapore (Singapore) • European Optical Society

\section{Supporting Organizations}

CAST_China Association for Science and Technology (China)

NSFC-National Nature Science Foundation (China)

Published by

SPIE

Volume 9274

Proceedings of SPIE 0277-786X, V. 9274

SPIE is an international society advancing an interdisciplinary approach to the science and application of light.

Advanced Sensor Systems and Applications VI, edited by Tiegen Liu, Shibin Jiang,

Niels Neumann, Proc. of SPIE Vol. 9274, 927401 - (c) 2014 SPIE

CCC code: $0277-786 X / 14 / \$ 18 \cdot$ doi: $10.1117 / 12.2181316$ 
The papers included in this volume were part of the technical conference cited on the cover and title page. Papers were selected and subject to review by the editors and conference program committee. Some conference presentations may not be available for publication. The papers published in these proceedings reflect the work and thoughts of the authors and are published herein as submitted. The publisher is not responsible for the validity of the information or for any outcomes resulting from reliance thereon.

Please use the following format to cite material from this book:

Author(s), "Title of Paper," in Advanced Sensor Systems and Applications VI, edited by Tiegen Liu, Shibin Jiang, Niels Neumann, Proceedings of SPIE Vol. 9274 (SPIE, Bellingham, WA, 2014) Article CID Number.

ISSN: 0277-786X

ISBN: 9781628413472

Published by

SPIE

P.O. Box 10, Bellingham, Washington 98227-0010 USA

Telephone +1 3606763290 (Pacific Time) · Fax +1 3606471445

SPIE.org

Copyright (C) 2014, Society of Photo-Optical Instrumentation Engineers.

Copying of material in this book for internal or personal use, or for the internal or personal use of specific clients, beyond the fair use provisions granted by the U.S. Copyright Law is authorized by SPIE subject to payment of copying fees. The Transactional Reporting Service base fee for this volume is $\$ 18.00$ per article (or portion thereof), which should be paid directly to the Copyright Clearance Center (CCC), 222 Rosewood Drive, Danvers, MA 01923. Payment may also be made electronically through CCC Online at copyright.com. Other copying for republication, resale, advertising or promotion, or any form of systematic or multiple reproduction of any material in this book is prohibited except with permission in writing from the publisher. The CCC fee code is 0277-786X/14/\$18.00.

Printed in the United States of America.

Publication of record for individual papers is online in the SPIE Digital Library.

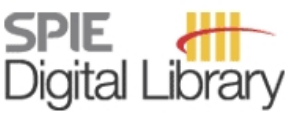

SPIEDigitalLibrary.org

Paper Numbering: Proceedings of SPIE follow an e-First publication model, with papers published first online and then in print and on CD-ROM. Papers are published as they are submitted and meet publication criteria. A unique, consistent, permanent citation identifier (CID) number is assigned to each article at the time of the first publication. Utilization of CIDs allows articles to be fully citable as soon as they are published online, and connects the same identifier to all online, print, and electronic versions of the publication. SPIE uses a six-digit CID article numbering system in which:

- The first four digits correspond to the SPIE volume number.

- The last two digits indicate publication order within the volume using a Base 36 numbering

system employing both numerals and letters. These two-number sets start with 00, 01, 02, 03, 04, $05,06,07,08,09,0 A, 0 B \ldots$. 0Z, followed by 10-1Z, 20-2Z, etc.

The CID Number appears on each page of the manuscript. The complete citation is used on the first page, and an abbreviated version on subsequent pages. Numbers in the index correspond to the last two digits of the six-digit CID Number. 


\title{
Contents
}

\author{
vii Authors \\ ix Symposium Committees \\ xi Conference Committee
}

SESSION 1 SENSOR SYSTEM/NETWORK AND MAGNETO-OPTIC SENSORS

927402 Novel approach for simultaneous wireless transmission and evaluation of optical sensors (Invited Paper) [9274-1]

927403 Magnetooptic sensor based on field-induced birefringence compensation [9274-2]

927404 A hybrid optic-fiber sensor network with the function of self-diagnosis and self-healing [9274-3]

\section{SESSION 2 DISTRIBUTED FIBER SENSORS}

927407 Long-range high spatial resolution optical frequency-domain reflectometry based on optimized deskew filter method (Invited Paper) [9274-6]

\section{SESSION 3 FIBER BRAGG GRATING AND WAVEGUIDE SENSORS I}

9274 OB Dual fiber Bragg gratings configuration-based fiber acoustic sensor for low-frequency signal detection [9274-11]

9274 OC Analysis on intensity demodulated strain sensing based on multiple phase-shiffed FBG [9274-12]

9274 OD Broadband-rejection filters using chirped and tilted fiber gratings [9274-13]

$9274 \mathrm{OE} \quad$ Strain transfer error analysis of optical fiber sensors with local debonding interface [9274-14]

9274 OF Packaged FBG sensors for real-time stress monitoring on deep-water riser [9274-15]

\section{SESSION 4 FIBER BRAGG GRATING AND WAVEGUIDE SENSORS II}

9274 OG Graphene-coated microfiber Bragg grating for ammonia gas sensing [9274-16]

$9274 \mathrm{OH} \quad$ Measurements of high frequency vibration using fiber Bragg grating sensors packaged on PZT plate [9274-17] 
92740 Ol Integrated Raman spectroscopic sensor based on silicon nanowire waveguides [9274-18]

9274 OJ High signal-to-noise acoustic sensor using phase-shifted gratings interrogated by the Pound-Drever-Hall technique [9274-19]

\section{SESSION 5 NEW TECHNIQUES, MATERIALS, AND STRUCTURES FOR OPTIC SENSORS}

9274 OK Very high-resolution optical spectral analysis based on Brillouin dynamic gratings (Invited Paper) [9274-20]

9274 OL High Q silica microbubble resonators fabricated by heating a pressurized glass capillary [9274-21]

9274 OM Research of AGC technology in a digital optical fiber sensing system with PGC modulation and demodulation [9274-22]

$927400 \quad$ Two kinds of novel tunable Thulium-doped fiber laser [9274-24]

9274 OP Micro-bubble-based wavelength division multiplex optical fluidic sensing [9274-25]

SESSION 6 FIBER SENSORS FOR VIBRATION, STRAIN, AND PRESSURE SENSING

9274 OR Measurement of high-intensity focused ultrasound fields using miniaturized all-silica fiber-optic Fabry-Perot hydrophones (Invited Paper) [9274-27]

9274 OS Sensing characteristics of clad-modified with nanocrystalline metal oxide fiber optic gas sensor [9274-36]

9274 OT Simultaneous measurement of acoustic pressure and temperature in the HIFU fields using all-silica fiber optic Fabry-Perot hydorophone [9274-28]

9274 OU A positioning algorithm based on super-resolution time delay estimation in dual Mach-Zehnder interferometry disturbance sensor [9274-29]

9274 OV Fiber optic pressure sensing method based on Sagnac interferometer [9274-30]

$9274 \mathrm{OW} \quad$ Health monitoring of electric power communication line using a distributed optical fiber sensor [9274-31]

9274 OX Application of optical fiber sensor based on optical vortex to high resolution strain measurement [9274-32]

POSTER SESSION

927412 The simulation for optimal design of metal film on surface plasmon resonance sensor [9274-38] 
927413 Simulation on the particle flow in laser airborne particle counting sensor [9274-39]

927414 Fiber-tip bubble-structure microcavity sensor [9274-40]

927415 Tunable mode coupler in the microfluidic channel for the fiber optics refractive index sensor [9274-41]

927416 Confocal ring cavity as micro-optical gyro element [9274-42]

927417 Simplified design of diaphragm-based fiber optic extrinsic Fabry-Perot accelerometer [9274-43]

927418 Fiber optical accelerometer based on 45 degrees Fabry-Perot cavity [9274-44]

927419 A novel porous silicon-based multilayer dielectric-grating structures for diffraction-based sensing [9274-45]

9274 1A Intensity modulation photonic crystal fiber based refractometer in the visible wavelength range [9274-46]

9274 1B Photonic crystal fiber refractive-index sensor based on multimode interferometry [9274-47]

9274 1C A new refractive index sensor based on Mach-Zehnder interferometer fabricated by two cascaded single-mode fiber corners [9274-48]

9274 ID The wavelength-tunable tapered surface plasmon resonance fiber sensor based on separated input-output channels [9274-49]

9274 IF Fabrication and simulation of cascaded Fabry-Perot micro-cavities in fibers [9274-51]

$9274 \mathrm{1H} \quad$ Long period fiber grating sensor in hollow eccentric optical fiber [9274-53]

$927411 \quad$ Characteristics of all-solid photonic crystal fibers with radial linear arrays [9274-54]

$92741 \mathrm{~K}$ Laser sensor for monitoring radioactive contamination [9274-56]

$92741 \mathrm{~L} \quad$ Laser remote spectroscopy for geological exploration of hydrocarbons deposits [9274-57]

9274 IM Development of a long-gauge vibration sensor [9274-58]

9274 iN The effect of linear birefringence on fiber optic current sensor based on Faraday mirror [9274-60]

927410 Vehicle self-velocimeter for navigation system based on a linear image sensor [9274-61]

9274 IP Fiber-optic temperature sensor based on specialty triple-clad fiber [9274-62]

9274 1Q Temperature characteristic of hollow-core photonic crystal fiber resonator [9274-63]

9274 IR Polydimethylsiloxane-fabricated optical fiber sensor capable of measuring both large axial and shear strain [9274-64] 
9274 is Application of pulse cavity ring-down spectroscopy technique for aerosol extinction measurement [9274-65]

9274 IV Numerical investigation of photonic crystal fiber sensor sensitivity based on evanescent wave absorption [9274-68]

$9274 \mathrm{IW} \quad$ Vectorial E-field sensing using pigtailed photonic probes for aperture-field mapping of antennas [9274-69]

$92741 \mathrm{X}$ Influence of laser linewidth on spectral ripple in fold-type cavity ring-down measurement [9274-70]

9274 IY Optical fiber voltage sensor based on Michelsion interferometer using Fabry-Perot demodulation interferometer [9274-71]

927412 A micro particle launching apparatus based on mode-division-multiplexing technology [9274-72]

927420 Birefringence characteristic research of $\mathbf{4 0}$ micron supersmall diameter elliptical cladding type polarization maintaining fiber [9274-73]

927423 Temperature and pressure measurement based on tunable diode laser absorption spectroscopy with gas absorption linewidth detection [9274-76]

927425 The effect of near-infrared laser beam on the surface modification of metal complex based on 3D laser scanning system [9274-78] 


\section{Authors}

Numbers in the index correspond to the last two digits of the six-digit citation identifier (CID) article numbering system used in Proceedings of SPIE. The first four digits reflect the volume number. Base 36 numbering is employed for the last two digits and indicates the order of articles within the volume. Numbers start with 00, 01, 02, 03, 04, 05, 06, 07, 08, 09, OA, OB...0Z, followed by 10-1Z, 20-2Z, etc.

Bai, Yang, OF

Bespalov, V. G., 1K, $1 \mathrm{~L}$

Bi, Weihong, IP

Cao, Fangfang, $1 \mathrm{Z}$

Chai, Tianjiao, OU

Chen, Cheng, 04

Chen, Daru, 00, 14, 1C

Chen, HuiBin, 12

Chen, Lin-Xun, OX

Chen, Qinnan, OU

Chen, Shimeng, 1A, 1D

Chen, Wenjie, OL, OP

Chen, Wentao, $\mathrm{OH}$

Chen, Xin-wei, IW, IY

Chen, Ying, OV

Comanici, Maria I., 0J, IM

Dai, Huanhuan, $1 \mathrm{C}$

Ding, Zhenyang, 07, OU

Dong, Yongkang, OK

Du, Yang, 07

Elizarov, V. V., 1K, 1L

Fan, Yan'en, $1 Y$

Fang, Qiaofeng, $\mathrm{OH}$

Feng, Bowen, 07

Feng, Gaofeng, 00, 14

Feng, Lishuang, $1 Q$

Filatov, Yuri $\vee ., 16$

Fu, Guangwei, 1P

Fu, Salai, 1C

Fu, Xinghu, $1 \mathrm{P}$

Gao, R., 15

Gao, Xiaotong, 1D

Ge, Chunfeng, 04

Gong, Yuan, OG

Gong, Zhenfeng, 1A, 1B

Grishkanich, A. S., 1K, $1 \mathrm{~L}$

Gu, Fan, $1 F$

Guan, Bai-Ou, OD

Guan, Chunying, 1H, 11

Guo, Jie, $1 S$

Guo, Jinghong, OW

Guo, Peng, 1P

Guo, Tuan, OD

Han, Jing, 17, 18

$\mathrm{He}$, Chang, OU

$\mathrm{He}$, Jian-Jun, Ol

$\mathrm{He}$, Shengnan, $1 \mathrm{Y}$

$\mathrm{He}$, Xin, 10

Huang, Jie, IV
Huang, Wenzhu, 17

Il'inskiy, A. A., $1 \mathrm{~L}$

Ji, Yunjing, 13

Jia, Ping-Gang, OR, OT

Jia, Zhenhong, 19

Jiang, Junfeng, 07, OL, OP, OU, 23, 25

Jiang, Taofei, OK

Jiang, $X ., 14$

Jiang, Xianxin, 0 I

Jiang, Xin-Yin, OR, OT

Jiang, $Y ., 15$

Jiang, Yajun, $\mathrm{OF}$

Jiao, Hongchen, $1 Q$

Kang, C., 20

Kascheev, S. V., 1K, $1 \mathrm{~L}$

$\mathrm{Ke}$, Ding, OR

Kung, Peter, OJ, $1 \mathrm{M}$

Lai, Jiancheng, 13

Li, Benchong, IV

$\mathrm{Li}$, Binglin, OW

Li, Changsheng, 03

Li, Dandan, IY

Li, Fang, 17, 18

Li, G., 15

Li, Lin, $1 \mathrm{~N}$

Li, Mingyu, Ol

Li, Qian, $1 M$

Li, Shujuan, OC

Li, Zhenhua, 13

Liang, Yun, OW

Lin, Xujun, OL

Liu, D. Q., 20

Liu, Deming, OB

Liu, Fu, OD

Liu, Kun, 07, OL, OP, OU, 23

Liu, Lei, OR, OT

Liu, Tiegen, 04, 07, OL, OP, OU, 1N, 23, 25

Liu, Wanqiu, OE

Liv, Wenhui, OL, OP

Liu, Xiuxin, 1D

Liu, Yajun, 19

Liu, Yun, 1A, 1B, 1D

Liu, Yunqi, $\mathrm{OH}$

Liu, Zhihai, $1 \mathrm{Z}$

Liu, Zigeng, 1B

Long, Xingwu, 10, 1X

Lou, Jun, IV

Lu, Lidong, OW

Lu, Ping, $O B$ 
LUo, S., 14

LV, Changwu, 19

Lv, Xiaoyi, 19

Ma, Chunyu, OU

Ma, Xiaowei, 00, 14

Mao, Guopei, $1 \mathrm{H}$

Meng, Yunxia, 23

Mo, Jiaqing, 19

Neumann, Niels, 02

$\mathrm{Ni}$, Jiasheng, $\mathrm{OC}$

Nie, Xiaoming, 10

Pan, Liang, OU

Pang, Fufei, $1 \mathrm{~F}$

Peng, Baojin, 1C

Peng, Gangding, OC

Peng, Wei, 1A, 1B, 1D

Plettemeier, Dirk, 02

Qi, Haifeng, OC

Rao, Yunjiang, $0 G$

Ren, Xiaoyuan, $1 Q$

Renganathan, B., OS

Sastikumar, D., OS

Schuster, Tobias, 02

Sevryugin, Alexander A., 16

Shalymov, Egor V., 16

Shao, Jie, is

Shen, Weimin, $1 \mathrm{~V}$

Shen, Yize, 11

Shen, YU, IR

Shi, Qingguo, 1C

Song, Jinyan, ol

Song, Wenshuai, 1Q

Song, Zhiqiang, $\mathrm{OC}$

Sun, Baochen, 18

Tan, Zhongqi, $1 \mathrm{X}$

Tang, Jianfeng, OM

Tang, Longhua, $\mathrm{Ol}$

Tian, S., 20

Vasiev, S. K., IK

Venediktov, Vladimir Yu., 16

Wang, Chang, OC

Wang, Chang-lei, IW

Wang, Chunyong, 13

Wang, Dai-Hua, OR, OT

Wang, Huaping, OE

Wang, Kai, IY

Wang, Liming, is

Wang, Meirong, OF

Wang, Meng, 25

Wang, Ranran, 23

Wang, Shun, OB

Wang, Tao, 23

Wang, Tingyun, $1 \mathrm{~F}$

Wang, Zhaogang, 17, 18

Wang, Ziyuan, $1 \mathrm{R}$

Wei, Wenjian, $1 \mathrm{X}$

Wen, Huaihai, $1 R$

Wen, Yaowu, 11

Wu, Shuai, IW, $1 Y$
Wu, Suyong, $1 x$

Wu, YU, OG

Xiang, Jindong, is

Xie, Haiyang, IP

Xiong, Jiabao, $1 \mathrm{~F}$

Xiong, Shuidong, OM

Xu, Ben, IV

$\mathrm{XU}$, Binhong, 18

$X \cup$, Hongzhi, $1 \mathrm{~V}$

$X U$, Jian, $O F$

$\mathrm{Xu}$, Shibo, 04

$\mathrm{XU}$, Yihang, 11

Yan, Wei, 13

Yang, Dexing, OF

Yang, Dong, OB

Yang, Haibo, 11

Yang, Jing, 11

Yang, Junyong, 00, 14

Yang, Li, OX

Yang, Yuanhong, OV

Yao, Baicheng, OG

Yao, X. Steve, 07, IN

Yin, Zhenyu, $\mathrm{OH}$

Yu, Zhe, OL, OP

Yuan, Li-Bo, 1H, 20

Yuan, Tingting, $1 \mathrm{H}$

Zeng, LU-YU, OR, OT

Zhai, Huailun, OF

Zhang, Anqi, OG

Zhang, Chao, IP

Zhang, Faxiang, OC

Zhang, Hao, OW

Zhang, Hongxia, 04

Zhang, Rongxiang, $1 \mathrm{~N}$

Zhang, T., 20

Zhang, Wentao, 17, 18

Zhang, Xiaobei, IF

Zhang, Xiaolei, OC

Zhang, Xinpu, 1A, 1B

Zhang, Xuezhi, OL, OP

Zhang, Xuping, OW

Zhang, Yan, $O M$

Zhang, Yi, OV

Zhang, Yiwei, $1 \mathrm{M}$

Zhao, Mali, 25

Zheng, Qingying, is

Zhevlakov, A. P., 1K, $1 \mathrm{~L}$

Zhong, Xing, $1 \mathrm{H}$

Zhou, Jian, 10

Zhou, Y., 15

Zhou, Zhen, is

Zhou, Zhi, OE, IR

Zhuang, Zhi, OV

Zou, Ting, 13 


\title{
Symposium Committees
}

\author{
General Chairs
}

H. Philip Stahl, NASA Marshall Space Flight Center (United States) Bingkun Zhou, Tsinghua University (China)

General Co-chairs

Arthur Chiou, National Yang-Ming University (Taiwan, China) Jianlin Cao, China Ministry of Science and Technology (China) Junhao Chu, Shanghai Institute of Technical Physics (China)

Technical Program Chairs

Songlin Zhuang, Shanghai University of Science and Technology (China)

Xingde Li, Johns Hopkins University (United States)

Technical Program Co-chairs

Qiming Wang, Institute of Semiconductors (China)

Xu Liu, Zhejiang University (China)

Daoyin Yu, Tianjin University (China)

Qihuang Gong, Peking University (China)

Tianchu Li, National Institute of Metrology (China)

Wei Huang, Nanjing University of Posts and Telecommunications (China)

Local Organizing Committee Chair

Guangcan Guo, University of Science and Technology of China (China) 
Local Organizing Committee Co-chairs

Guoqiang Ni, Beijing Institute of Technology (China)

Shusen Xie, Fujian Normal University (China)

Xiaomin Ren, Beijing University of Posts and Telecommunications (China)

Ying Gu, People's Liberation Army General Hospital (China)

Huilin Jiang, Changchun University of Science and Technology (China)

General Secretary

Qihuang Gong, Peking University (China)

Local Organizing Committee

Yan Li, Chinese Optical Society/Peking University (China)

Zhiping Zhou, Peking University (China)

Changhe Zhou, Shanghai Institute of Optics and Fine Mechanics (China)

Qingming Luo, Huazhong University of Science and Technology (China)

Chongxiu Yu, Beijing University of Posts and Telecommunications (China)

Hongda Chen, Institute of Semiconductors (China)

Yongtian Wang, Beijing Institute of Technology (China)

Yiping Cui, Southeast University (China)

Xuping Zhang, Nanjing University (China)

Feijun Song, Daheng Corporation (China)

Cunlin Zhang, Capital Normal University (China)

Yanting Lu, Nanjing University (China)

Yuejin Zhao, Beijing Institute of Technology (China)

Chunqing Gao, Beijing Institute of Technology (China)

Tiegen Liu, Tianjin University (China)

Xiaocong Yuan, Nankai University (China)

Weimin Chen, Chongqing University (China)

Zhongwei Fan, Academy of Optoelectronics (China)

Hanyi Zhang, Tsinghua University (China)

Lan Wu, Zhejiang University (China)

Yongsheng Zhang, University of Science and Technology of China (China)

Hong Yang, Peking University (China)

Xiaoying Li, Tianjin University (China)

Wei Xiong, Chinese Optical Society (China) 


\title{
Conference Committee
}

\author{
Conference Chairs
}

Tiegen Liu, Tianjin University (China)

Shibin Jiang, AdValue Photonics, Inc. (United States)

Niels Neumann, Technische Universität Dresden (Germany)

Conference Program Committee

Xiaoyi Bao, University of Ottawa (Canada)

Rongshen Chen, The University of Birmingham (United Kingdom)

Kin-Seng Chiang, City University of Hong Kong (Hong Kong, China)

Brian Culshaw, University of Strathclyde (United Kingdom)

Xudong Fan, University of Michigan (United States)

Claire Gu, University of California, Santa Cruz (United States)

Baiou Guan, Jinan University (China)

Zuyuan He, Shanghai Jiao Tong University (China)

Wei Jin, The Hong Kong Polytechnic University Shenzhen Research Institute (China)

Rene Landgraf, Fraunhofer-Institut für Photonische Mikrosysteme (Germany)

Deming Liu, Huazhong University of Science and Technology (China)

Li Pei, Beijing Jiaotong University (China)

Gang-Ding Peng, The University of New South Wales (Australia)

Xueguang Qiao, Northwest University (China)

Yunjiang Rao, University of Electronic Science and Technology of China (China)

Tobias Schuster, Technische Universität Dresden (Germany)

Anbo Wang, Virginia Polytechnic Institute and State University (United States)

Tingyun Wang, Shanghai University (China)

Hai Xiao, Clemson University (United States)

Steve Yao, General Photonics Corporation (United States)

Paul Kit-Lai Yu, University of California, San Diego (United States)

Li-Bo Yuan, Harbin Engineering University (China)

\section{Session Chairs}

1 Sensor System/Network and Magneto-Optic Sensors

Steve Yao, General Photonics Corporation (United States)

2 Distributed Fiber Sensors

Niels Neumann, Technische Universität Dresden (Germany) 
3 Fiber Bragg Grating and Waveguide Sensors I

Yongkang Dong, Harbin Institute of Technology (China)

4 Fiber Bragg Grating and Waveguide Sensors II

Shibin Jiang, AdValue Photonics, Inc. (United States)

5 New Techniques, Materials, and Structures for Optic Sensors

Tuan Guo, Jinan University (China)

6 Fiber Sensors for Vibration, Strain, and Pressure Sensing

Tiegen Liu, Tianjin University (China) 